\title{
Mass zeros in the one-loop effective actions of QED in $1+1$ and $3+1$ dimensions
}

\author{
M. P. Fry * \\ School of Mathematics, University of Dublin, \\ Dublin 2, Ireland
}

\begin{abstract}
It is known that the one-loop effective action of $\mathrm{QED}_{2}$ is a quadratic in the field strength when the fermion mass is zero: all potential higher order contributions beyond second order vanish. For nonzero fermion mass it is shown that this behavior persists for a general class of fields for at least one value of the fermion mass when the external field's flux $\Phi$ satisfies $0<$ $|e \Phi|<2 \pi$. For $\mathrm{QED}_{4}$ with (anti-) self-dual fields the mass-shell renormalized one-loop effective action vanishes for at least one value of the fermion mass provided a plausible zero-mass limit exists.
\end{abstract}

PACS numbers: 12.20.Ds, 11.10.Kk, 11.15Tk

*Electronic mail: mpfry@maths.tcd.ie 


\section{INTRODUCTION}

In all gauge field theories coupled to fermions the fermionic determinant is fundamental. These determinants, denoted by det, produce an effective functional measure for the gauge fields when the fermionic fields are integrated. The continuing lack of nonperturbative information on these determinants is reflected in the necessity to make loop expansions or the more extreme quenched approximation in which the determinant is ignored. Nonperturbative approaches such as Monte Carlo evaluations with a discrete lattice regulator result in algorithms that currently dominate this area. Most analytic nonperturbative results obtained so far deal with the dependence of the determinants on the coupling constant. Little attention has been given to their dependence on the fermion mass. Here we will confine our attention to quantum electrodynamics in two and four dimensions in the belief that any progress made might suggest how to proceed in other cases. Furthermore, nonperturbative QED is of interest in its own right.

It might be objected that $\mathrm{QED}_{2}$ is of no physical interest, certainly not the mass dependence of its fermionic determinant. This is not true. Firstly, when the Wick rotation to Euclidean space is made, $\operatorname{det}_{\mathrm{QED}_{2}}$ is calculated from the eigenfunctions of the two-dimensional Pauli operator $(P-A)^{2}-\sigma_{3} B$ in a magnetic field $B$ normal to a plane. In what follows the coupling constant $e$ is assumed to be absorbed by the potential $A_{\mu}$. Then $\operatorname{det}_{\mathrm{QED}_{2}}$ fully determines $\operatorname{det}_{\mathrm{QED}_{3}}$ [1] and $\operatorname{det}_{\mathrm{QED}_{4}}$ [2] for the same magnetic field, namely

$$
\begin{gathered}
\frac{\partial}{\partial m^{2}} \ln \operatorname{det}_{\mathrm{QED}_{4}}=-\frac{L^{2}}{2 \pi} \ln \operatorname{det}_{\mathrm{QED}_{2}}-\frac{L^{2}\|B\|^{2}}{24 \pi^{2} m^{2}}, \\
\ln \operatorname{det}_{\mathrm{QED}_{3}}=\frac{L}{2 \pi} \int_{m^{2}}^{\infty} \frac{d M^{2}}{\sqrt{M^{2}-m^{2}}} \ln \operatorname{det}_{\mathrm{QED}_{2}}\left(M^{2}\right),
\end{gathered}
$$

where $L$ is the edge length of a space-time box and $\|B\|^{2}=\int d^{2} x B^{2}(x)$. Equation (1.1) assumes mass-shell change renormalization while (1.2) assumes a $2 \times 2$ representation of the Dirac $\gamma$-matrices. By continuing back to Minkowski space these equations give the effective action $i S=\ln$ det for a two-variable static magnetic field in $2+1$ and $3+1$ dimensions.

Secondly, suppose $\operatorname{det}_{\mathrm{QED}_{2}}$ is calculated for the single-variable magnetic field $B=$ $B_{0} f(x / \lambda)$. The duality transformation $B \rightarrow e^{-i \pi / 2} E$, where $E=E_{0} f(t / \tau)$ and $\tau=i \lambda$, gives the pair nonproduction probability $e^{-2 \operatorname{Im} S}$ with $\operatorname{Im} S^{3+1}$ and $\operatorname{Im} S^{2+1}$ obtained from 
(1.1) and (1.2). Duality in this restricted sense has been demonstrated recently by Dunne and Hall [3, 团. Conditions for the validity of the more general duality transformation $B(x, y) \rightarrow e^{-i \pi / 2} E(x, t)$ are unknown.

There are no exact calculations of $S$ in any dimension for two-variable fields $B(x, y)$ or $E(x, t)$, or even finite-flux magnetic fields, except for the two-dimensional case of a magnetic field confined to the wall of a cylinder [5]. Actions for slowly varying fields can be calculated in a derivative expansion [6 [10]. For more general fields semiclassical estimates of $S$ are effective provided the analysis can be carried through [3,11]. So far this has limited the background field to a dependence on a single space or time variable, effectively special cases of $\mathrm{QED}_{2}$.

The Euclidean $\mathrm{QED}_{2}$ determinant can be expressed as [2,12 [14]

$$
\ln \operatorname{det}_{\mathrm{QED}_{2}}=-\frac{1}{2 \pi} \int \frac{d^{2} k}{(2 \pi)^{2}}|\hat{B}(k)|^{2} \int_{0}^{1} d z \frac{z(1-z)}{k^{2} z(1-z)+m^{2}}+\ln \operatorname{det}_{3},
$$

where $\hat{B}$ is the Fourier transform of $\mathrm{B}$ and $\ln \operatorname{det}_{3}$ may for the present be viewed as the sum of all one-loop fermion graphs, beginning in fourth order. It is gauge invariant, depending only on $B$. It is known that $\ln \operatorname{det}_{3}\left(m^{2}=0\right)=0$. This was first shown by Schwinger [15]. Seiler [12 later gave a compact proof of Schwinger's result and stated the precise condition for it to be true, namely $A_{\mu} \in L^{n}\left(\mathbb{R}^{2}\right), n>2$. Furthermore,

$$
\lim _{m^{2}=0} \ln \operatorname{det}_{3}\left(m^{2}\right)=0
$$

provided the magnetic field's flux $\Phi=0$. This result requires several nontrivial estimates from analysis and will be published elsewhere. But it is plausible: if $\hat{B}(0)=0$ or equivalently, $\Phi=0$, then the infrared properties of $\ln \operatorname{det}_{3}$ are improved, allowing continuity at $m^{2}=0$. In Sec. II we will show that for potentials $A_{\mu} \in L^{n}\left(\mathbb{R}^{2}\right), n>2$ and finite-range magnetic fields with $B \in L^{n}\left(\mathbb{R}^{2}\right), n=2,4$ and $\int d^{2} x B^{2}\left(\partial_{\mu} B\right)^{2}<\infty$ there is at least one value of $m^{2}>0$ for which $\ln \operatorname{det}_{3}=0$, provided $0<|\Phi|<2 \pi$. Therefore, our result is this: when $0<|\Phi|<2 \pi$ the zero in $m^{2}$ of $\ln \operatorname{det}_{3}$ moves up from $m^{2}=0$ when $\Phi=0$ to some finite value(s) $m^{2}>0$. For $|\Phi| \geq 2 \pi$ our analysis is unable to say anything about the zeros in $m^{2}$ of $\ln _{\operatorname{det}_{3}}$. Apparently their presence or absence is tied in with the formation of square-integrable zero modes of the two-dimensional Pauli operator when $|\Phi|>2 \pi[16]$. 
The presence of zeros in $m^{2}$ in $\ln \operatorname{det}_{3}$ together with the result [17] that ln $\operatorname{det}_{3}$ is bounded above and below by terms quadratic in $B$ suggest that ln $\operatorname{det}_{3}$ is small in the sense that it is comparable to the second-order term in (1.3). These bounds are obtained from Eq.(9) in 17 and the definition (1.3) above. The lower bound on ln det $_{3}$ from [17] has been established for fields $B \geq 0$ or $\leq 0$ over all space, a technicality that a better estimate might overcome.

In Sec. III we establish the conditions for the large $m^{2}$ expansion of $\ln \operatorname{det}_{3}$ to be an asymptotic series, a result required in Sec. II and useful in Sec. IV.

For Euclidean $\mathrm{QED}_{4}$ we will present evidence in Sec. IV that ln $\operatorname{det}_{\mathrm{QED}_{4}}$ vanishes for at least one value of $m^{2}$ for non-constant self-dual (anti-self-dual) finite-range fields $\mathbf{B}=-\mathbf{E}$ $(\mathbf{B}=\mathbf{E})$ provided $A_{\mu} \in L^{n}\left(\mathbb{R}^{4}\right), n>4$ and the integrals of $\left(\partial_{\mu} B\right)^{2},\left(\partial_{\mu} \partial_{\nu} B\right)^{2}, B^{4}$ and $B^{6}$ over $\mathbb{R}^{4}$ are finite. We are restricted to such fields in the absence of a theorem in four dimensions, analogous to the Aharonov-Casher theorem in two dimensions [16], that counts the total number of zero modes for general fields. Our result is tentative as it requires the proof of the limit in (4.8) below. We believe (4.8) can be proved, thereby validating new nonperturbative information on $\mathrm{QED}_{4}$.

\section{TWO-DIMENSIONAL QED}

The fermionic determinant in Euclidean QED is defined here by Schwinger's [18] heat kernel representation

$$
\ln \operatorname{det}=\frac{1}{2} \int_{0}^{\infty} \frac{d t}{t}\left\{\operatorname{Tr}\left(e^{-P^{2} t}-\exp \left[-\left(D^{2}+\frac{1}{2} \sigma F\right) t\right]\right)+\frac{\|F\|^{2}}{24 \pi^{2}}\right\} e^{-t m^{2}} .
$$

Here $D^{2}=(P-A)^{2}, \sigma^{\mu \nu}=\left[\gamma^{\mu}, \gamma^{\nu}\right] / 2 i, \gamma^{\mu \dagger}=-\gamma^{\mu}$, and $\|F\|^{2}=\int d^{4} x F_{\mu \nu}^{2}$. The last term in (2.1) is the second-order mass-shell charge renormalization subtraction required for the small $t$ limit of the integral to converge. In two and three dimensions this term should be omitted. If $-\ln$ det is combined with the Maxwell action to form an effective measure for $A_{\mu}$ then $A_{\mu}$ has to be concentrated on $\mathcal{S}^{\prime}$, the Schwartz space of tempered distributions. Such potentials have to be temporarily smoothed until after the integration over $A_{\mu}$ if sense is to be made of the right-hand side of (2.1). This procedure has been discussed elsewhere [1,5]. Here we will simply assume that $A_{\mu}$ and $F_{\mu \nu}$ are sufficiently smooth and fall off rapidly enough for our analysis to go through. More specific statements will be made below. 
Specializing to $\mathrm{QED}_{2}$ and expanding the right-hand side of (2.1) to second order gives the standard perturbative result in the first term in (1.3). The remainder, $\ln \operatorname{det}_{3}$, is given by (3.2) below. In coordinate space (1.3) is

$$
\ln \operatorname{det}_{\mathrm{QED}_{2}}=\int d^{2} x d^{2} y B(x) \Pi(x-y) B(y)+\ln \operatorname{det}_{3},
$$

where

$$
\begin{aligned}
\Pi(x) & =-\frac{1}{2 \pi} \int \frac{d^{2} k}{(2 \pi)^{2}} e^{i k x} \int_{0}^{1} d z \frac{z(1-z)}{k^{2} z(1-z)+m^{2}} \\
& =-\frac{1}{4 \pi^{2}} \int_{0}^{1} d z K_{0}\left(\frac{|m x|}{\sqrt{z(1-z)}}\right)
\end{aligned}
$$

Assuming that $\mathrm{B}$ has finite range, the $m^{2} \rightarrow 0$ limit can be interchanged with the $x$ and $y$ integrals in (2.2), giving

$$
\int d^{2} x d^{2} y B(x) \Pi(x-y) B(y) \underset{m^{2} \rightarrow 0}{=} \frac{\Phi^{2}}{8 \pi^{2}} \ln m^{2}+O(1)
$$

We have shown that if $B$ is square integrable and has finite range then [19

$$
\ln \operatorname{det}_{\mathrm{QED}_{2}} \underset{m^{2} \rightarrow 0}{=} \frac{|\Phi|}{4 \pi} \ln m^{2}+R\left(m^{2}\right)
$$

where $\lim _{m^{2}=0}\left[R\left(m^{2}\right) / \ln m^{2}\right]=0$. That $\ln \operatorname{det}_{\mathrm{QED}_{2}}$ is negative is a reflection of the paramagnetic property of charged fermions whereby the eigenvalues of the Pauli operator are on average lower relative to those of the free Hamiltonian $P^{2}$ in the definition (2.1) 20,22]. The mass singularity in $\ln \operatorname{det}_{\mathrm{QED}_{2}}$ at $m^{2}=0$ is due to the formation of square-integrable zero modes and zero-energy unbound resonances in the continuum part of the Pauli operator's spectrum. The difference between (2.4) and (2.5) makes the nonperturbative nature of the result (2.5) evident. Equations (2.2), (2.4) and (2.5) give

$$
\ln \operatorname{det}_{3} \underset{m^{2} \rightarrow 0}{=} \frac{|\Phi|}{4 \pi}\left(1-\frac{|\Phi|}{2 \pi}\right) \ln m^{2}+R\left(m^{2}\right)
$$

from which one infers that $\ln \operatorname{det}_{3}<0$ if $0<|\Phi|<2 \pi$ and $m^{2}$ is sufficiently small.

The second piece of nonperturbative information required is 


$$
\ln \operatorname{det}_{3} \underset{m^{2} \rightarrow \infty}{=} \frac{1}{90 \pi m^{6}} \int d^{2} x B^{4}+\mathcal{R}\left(m^{2}\right),
$$

where

$$
\lim _{m^{2} \rightarrow \infty} m^{6} \mathcal{R}\left(m^{2}\right)=0
$$

This will be shown in Sec. III. It shows that for sufficiently large $m^{2} \ln \operatorname{det}_{3}$ becomes positive before approaching zero. This establishes our claim that $\ln \operatorname{det}_{3}$ has at least one zero for $m^{2}>0$ when $0<|\Phi|<2 \pi$.

\section{LARGE MASS BEHAVIOR OF DET ${ }_{3}$}

Here we will demonstrate (2.7) and (2.8). Integration over the fermions produces the formal result $\operatorname{det}(\not P-\not A+m) / \operatorname{det}(\not P+m)$. Another formal operation reduces this to $\operatorname{det}(1-S A)$, where $S=(P+m)^{-1}$. Because neither $S \not A$ nor $(S \not A)^{2}$ are trace class while $(S \not A)^{3}$ is in $\mathrm{QED}_{2}$ (see below) the identity $\ln \operatorname{det}(1+A)=\operatorname{Tr} \ln (1+A)$ for trace class operators has to be modified to

$$
\ln \operatorname{det}_{3}(1-S \not A)=\operatorname{Tr}\left[\ln (1-S \not A)+S \not A+\frac{1}{2}(S \not A)^{2}\right] .
$$

The right-hand side of (3.1) is the standard definition of a regularized determinant [13, 23-26]. Since $\operatorname{Tr}(S A)^{3}=0$ by Furry's theorem, the first nonvanishing term in (3.1) begins in fourth order. This leaves the second-order term in ln $\operatorname{det}_{\mathrm{QED}_{2}}$ to be defined by expanding definition (2.1) to second order, giving (1.3). Subtracting the second-order term from the heat kernel representation (2.1) of $\ln \operatorname{det}_{\mathrm{QED}_{2}}$ gives a definition of $\ln \operatorname{det}_{3}$ equivalent to (3.1) [13]:

$$
\begin{aligned}
\ln \operatorname{det}_{3}= & \frac{1}{2} \int_{0}^{\infty} \frac{d t}{t}\left\{\operatorname{Tr}\left(e^{-P^{2} t}-\exp \left[-\left(D^{2}+\frac{1}{2} \sigma F\right) t\right]\right)\right. \\
& \left.+\frac{t}{2 \pi} \int_{0}^{1} d z z(1-z) \int \frac{d^{2} k}{(2 \pi)^{4}}\left|\hat{F}_{\mu \nu}(k)\right|^{2} e^{-k^{2} z(1-z) t}\right\} e^{-t m^{2}}
\end{aligned}
$$

It was stated above that $(S A)^{3}$ is trace class in two dimensions. This follows from the result [26,27] that the operator $S(P) \not A(X)$ is a bounded operator on $L^{2}\left(\mathbb{R}^{2}\right)$ in the trace ideal $\mathcal{C}_{n}, n>2$ and 


$$
\|S(P) \not A(X)\|_{n} \leq\|S\|_{L^{n}}\|\not A\|_{L^{n}}
$$

Here $\mathcal{C}_{n}=\left\{A \mid\|A\|_{n}^{n}=\operatorname{Tr}\left(A^{\dagger} A\right)^{n / 2}<\infty\right\}$. By inspection $\|S\|_{L^{n}}<\infty$ for $n>2$. We hereafter assume that $A_{\mu} \in L^{n}\left(\mathbb{R}^{2}\right), n>2$, which is compatible with the $1 / r$ fall off of $A_{\mu}$ in the gauge $\partial_{\mu} A^{\mu}=0$ when $\Phi \neq 0$. Since $S \not A \in \mathcal{C}_{2+\epsilon}$ it belongs to all $\mathcal{C}_{n}$ with $n>2$, thus establishing our statement that $(S A)^{3}$ is trace class in two dimensions.

In the coordinate space representation of $S(P) \not A(X)$ the propagator is given by

$$
S(x)=\frac{1}{2 \pi}\left(\sqrt{m^{2}}+i \not \supset\right) K_{0}\left(\sqrt{m^{2} x^{2}}\right) .
$$

Hence $S$ is an analytic function of $m^{2}$ throughout the complex $m^{2}$-plane cut along the negative real axis. Then the following theorem of Gohberg and Krein [28] applies: Let $A(\mu) \in \mathcal{C}_{1}$ and be analytic in $\mu$ in some region. Then the $\operatorname{determinant} \operatorname{det}(1-A(\mu))$ is analytic in $\mu$ in the same region. In our case $S A \in \mathcal{C}_{2+\epsilon}$, requiring the two subtractions in (3.1). These subtractions can be easily incorporated into Gohberg and Krě̌n's proof for $S A \in \mathcal{C}_{1}$, provided use is made of the inequality [23,25]

$$
\left|\operatorname{det}_{n}(1+A)\right| \leq e^{\Gamma_{n}\|A\|_{n}^{n}},
$$

if $A \in \mathcal{C}_{n}$ and $\Gamma_{n}$ is a constant. Therefore, $\operatorname{det}_{3}(1-S A)$ is infinitely differentiable in $m^{2}$ on the open interval $(0, \infty)$. In addition, $\operatorname{det}_{3}$ has no zeros for $m^{2}>0$ and for real coupling. This was proved in Sec. III C of [1] for the case of $\operatorname{det}_{4}$ in three dimensions; the case of $\operatorname{det}_{3}$ in two dimensions follows immediately from this proof. The regulated determinant, $\ln \operatorname{det}_{n}$, is analogous to (3.1) with $n-1$ subtractions. Hence, $\ln \operatorname{det}_{3}$ is also infinitely differentiable in $m^{2}$ on $(0, \infty)$.

Next, we require a theorem of Ford [29]: Let $f(x)$ be an infinitely differentiable function of $x$ on $(a, \infty)$ and let $\phi(x)=f(1 / x)$. If the limits $\phi(+0), \phi^{\prime}(+0), \ldots$ exist then for $x$ on $(a, \infty)$

$$
f(x) \sim a_{0}+a_{1} / x+\cdots,
$$

with $a_{k}=\phi^{(k)}(+0) / k !, k=0,1, \ldots$ The series (3.6) is asymptotic in the sense that

$$
\lim _{x \rightarrow \infty} x^{n}\left[f(x)-\left(a_{0}+a_{1} / x+\cdots+a_{n} / x^{n}\right)\right]=0
$$


for $n=0,1, \ldots$

Now consider the asymptotic expansion of $\ln \operatorname{det}_{3}$ for large $m^{2}$. Referring to (3.2), this can be obtained from the high-temperature expansion

$$
\begin{aligned}
\operatorname{Tr}\left(e^{-\left[D^{2}+\frac{1}{2} \sigma F\right] t}-e^{-P^{2} t}\right)= & \frac{1}{4 \pi t} \int d^{2} x\left[\frac{2}{3} t^{2} B^{2}+\frac{2}{15} t^{3} B \partial^{2} B+t^{4}\left(\frac{1}{70} B \partial^{4} B-\frac{2}{45} B^{4}\right)\right. \\
& \left.+t^{5}\left(\frac{4}{63} B^{2} \partial_{\mu} B \partial^{\mu} B+\frac{1}{945} B \partial^{6} B\right)+\cdots\right]
\end{aligned}
$$

The terms of $O\left(B^{2}\right)$ are an easy consequence of second-order perturbative theory; the term $-2 t^{4} B^{4} / 45$ is an immediate consequence of the Euler-Heisenberg result specialized to two dimensions [18, 30, and the term $4 t^{5} B^{2} \partial_{\mu} B \partial^{\mu} B / 63$ follows from the results in [7, 10, again specialized to two dimensions. As previously noted, the second-order terms in (3.8), when substituted into (3.2), will be canceled by the counterterm, giving

$$
\ln \operatorname{det}_{3}=\frac{1}{90 \pi m^{6}} \int d^{2} x B^{4}-\frac{1}{21 \pi m^{8}} \int d^{2} x B^{2} \partial_{\mu} B \partial^{\mu} B+\cdots .
$$

Note that as the expansion continues there are integrals over increasing derivatives and powers of $B$. The finiteness of the coefficients of increasing powers of $1 / m^{2}$ requires additional conditions on $B$. Since the remainder after summing $n$ terms in an asymptotic series is of the order of the first neglected term [29] we must impose the additional conditions $\int d^{2} x B^{4}<\infty$ and $\int d^{x} B^{2} \partial_{\mu} B \partial^{\mu} B<\infty$ if the series in (3.9) is terminated at the first term. We have now satisfied the conditions of Ford's theorem, thereby establishing (2.7) and (2.8).

\section{FOUR DIMENSIONAL QED}

In a representation in which $\gamma_{5}$ takes the diagonal form $\gamma_{5}=\left(\begin{array}{cc}1 & 0 \\ 0 & -1\end{array}\right)$ the operator $-\not D^{2}$ is

$$
D^{2}+\frac{1}{2} \sigma F=\left(\begin{array}{cc}
H_{+} & 0 \\
0 & H_{-}
\end{array}\right)
$$

where

$$
H_{ \pm}=(P-A)^{2}-\boldsymbol{\sigma} \cdot(\mathbf{B} \pm \mathbf{E})
$$


Integration of the divergence of the axial current yields the global anomaly for $\not D$ in the form

$$
\frac{1}{4 \pi^{2}} \int d^{4} x \mathbf{E} \cdot \mathbf{B}(x)=m^{2} \operatorname{Tr}\left[\left(H_{+}+m^{2}\right)^{-1}-\left(H_{-}+m^{2}\right)^{-1}\right]
$$

with ${ }^{*} F^{\mu \nu}=\frac{1}{2} \epsilon^{\mu \nu \alpha \beta} F_{\alpha \beta}, \epsilon^{0123}=1, F^{k 0}=E^{k}$, and $F^{i j}=\epsilon^{i j k} B^{k}$. The $m^{2}=0$ limit of (4.3) gives a generalization of the Atiyah-Singer index theorem [31] to noncompact manifolds [32],

$$
\frac{1}{4 \pi^{2}} \int d^{4} x \mathbf{E} \cdot \mathbf{B}(x)=n_{+}-n_{-}+\frac{1}{\pi} \sum_{l} \mu(l)\left[\delta_{+}^{l}(0)-\delta_{-}^{l}(0)\right]
$$

where $n_{ \pm}$are the number of square-integrable zero modes of $H_{ \pm} ; \delta_{ \pm}^{l}(0)$ are the scattering phase shifts for $H_{ \pm}$as the energy tends to zero; $l$ is a degeneracy parameter, and $\mu(l)$ is a weight factor. By inspection of (4.2), self-dual (anti-self dual) fields $\mathbf{B}=-\mathbf{E}(\mathbf{B}=\mathbf{E})$ will have only negative (positive) chirality square-integrable zero modes. Suppose we choose $\mathbf{B}=\mathbf{E}$.

Differentiating the definition (2.1) of $\ln \operatorname{det}_{\mathrm{QED}_{4}}$ in the representation (4.1) and (4.2) gives

$$
\begin{aligned}
m^{2} \frac{\partial}{\partial m^{2}} \ln \operatorname{det}_{\mathrm{QED}_{4}=} & \frac{1}{2} m^{2} \operatorname{Tr}\left[\left(H_{+}+m^{2}\right)^{-1}-\left(H_{-}+m^{2}\right)^{-1}\right] \\
& +2 m^{2} \operatorname{Tr}\left[\left(D^{2}+m^{2}\right)^{-1}-\left(P^{2}+m^{2}\right)^{-1}\right]-\frac{1}{48 \pi^{2}}\|F\|^{2},
\end{aligned}
$$

where the second trace in (4.5) is defined as

$$
\operatorname{Tr}\left[\left(D^{2}+m^{2}\right)^{-1}-\left(P^{2}+m^{2}\right)^{-1}\right] \equiv \int_{0}^{\infty} d t e^{-t m^{2}} \operatorname{Tr}\left(e^{-D^{2} t}-e^{-P^{2} t}\right)
$$

consistent with definition (2.1); it is over space indices only. From (4.3) and the assumption $\mathbf{B}=\mathbf{E},(4.5)$ reduces to

$$
m^{2} \frac{\partial}{\partial m^{2}} \ln \operatorname{det}_{\mathrm{QED}_{4}}=\frac{1}{24 \pi^{2}}\|B\|^{2}+2 m^{2} \operatorname{Tr}\left[\left(D^{2}+m^{2}\right)^{-1}-\left(P^{2}+m^{2}\right)^{-1}\right] .
$$

If

$$
\lim _{m^{2} \rightarrow 0} m^{2} \operatorname{Tr}\left[\left(D^{2}+m^{2}\right)^{-1}-\left(P^{2}+m^{2}\right)^{-1}\right]=0
$$

then for $m^{2} \rightarrow 0$ 


$$
\ln \operatorname{det}_{\mathrm{QED}_{4}}=\frac{1}{24 \pi^{2}}\|B\|^{2} \ln m^{2}+R\left(m^{2}\right),
$$

where $\lim _{m^{2} \rightarrow 0}\left[R\left(m^{2}\right) / \ln m^{2}\right]=0$.

Is (4.8) true? In two dimensions with $\Phi>0$ the square-integrable zero modes of $H_{ \pm}=$ $(P-A)^{2} \mp B$ are confined to the positive chirality sector [16]. We then demonstrated [19] that $H_{-}$has the property

$$
\lim _{m^{2} \rightarrow 0} m^{2} \int_{0}^{\infty} d t e^{-t m^{2}} \operatorname{Tr}\left(e^{-\left(D^{2}+B\right) t}-e^{-P^{2} t}\right)=0
$$

Even if $\Phi$ is positive, $B$ can fluctuate in sign. We found that the integral in (4.10) only developed $\ln m^{2}$ type singularities as $m^{2} \rightarrow 0$. In four dimensions we choose $\mathbf{B}=\mathbf{E}$ to be certain that the zero modes are in the positive chirality sector. Then the dangerous fluctuating $B$-term is absent in the negative chirality sector. This and the tendency for infrared divergences to be less severe in higher dimensions lead us to conjecture that (4.8) is true. In the case of self-dual fields, $H_{+}$and $H_{-}$are interchanged in the above analysis. Thus, in both cases (4.9) is true if (4.8) is true. Then (4.9) indicates that ln $\operatorname{det}_{\mathrm{QED}_{4}}$ becomes negative as $m^{2} \rightarrow 0$, which is a reflection of paramagnetism [22].

Now consider the large mass behavior of $\ln \operatorname{det}_{\mathrm{QED}_{4}}$. In this case $S A \in \mathcal{C}_{d}, d>4$ provided $A_{\mu} \in L^{n}\left(\mathbb{R}^{4}\right), n>4$ so that 12 14

$$
\begin{aligned}
\ln \operatorname{det}_{\mathrm{QED}_{4}}= & \frac{1}{8 \pi^{2}} \int \frac{d^{4} k}{(2 \pi)^{4}}\left|\hat{F}_{\mu \nu}(k)\right|^{2} \int_{0}^{1} d z z(1-z) \ln \left(\frac{z(1-z) k^{2}+m^{2}}{m^{2}}\right) \\
& +\int \Pi_{\mu \nu \lambda \sigma} A_{\mu} A_{\nu} A_{\lambda} A_{\sigma}+\ln \operatorname{det}_{5}(1-S \not A) .
\end{aligned}
$$

The first two terms in (4.11) are obtained from the definition (2.1) by expanding it through fourth order. The fourth-order term in (4.11) has been dealt with explicitly by Karplus and Neuman [33]. Inspection of their result continued to Euclidean space shows that it is analytic in the complex $\mathrm{m}^{2}$-plane cut along the negative real axis. By Ford's theorem [29] it has an asymptotic expansion in $1 / \mathrm{m}^{2}$, whose leading term is [33]

$$
\int \Pi_{\mu \nu \lambda \sigma} A_{\mu} A_{\nu} A_{\lambda} A_{\sigma}=\frac{1}{2880 m^{4}} \int d^{4} x\left[14 F_{\mu \nu} F_{\nu \alpha} F_{\alpha \beta} F_{\beta \mu}-5\left(F_{\mu \nu} F_{\mu \nu}\right)^{2}\right]+\cdots
$$

The remainder term, ln $\operatorname{det}_{5}$, in (4.11) is like $\ln \operatorname{det}_{3}$ in (3.1) except that it has four subtractions: 


$$
\ln \operatorname{det}_{5}(1-S \not A)=\operatorname{Tr}\left[\ln (1-S \not A)+\sum_{n=1}^{4}(S \not A)^{n} / n\right]
$$

In the coordinate space representation of $S \not A$ the propagator is

$$
S(x)=\frac{m^{2}}{4 \pi^{2}}\left(\sqrt{m^{2}}+\not \partial\right)\left(K_{1}\left(\sqrt{m^{2} x^{2}}\right) / \sqrt{m^{2} x^{2}}\right),
$$

which is analytic in $m^{2}$ throughout the complex $m^{2}$-plane cut along the negative real axis. Therefore, the same analysis as in Sec. [III establishes that $\operatorname{det}_{5}$ is infinitely differentiable in $m^{2}$ on the interval $(0, \infty)$ provided use is made of (3.5) for $n=5$ to extend Gohberg and Krěn's theorem to det $_{5}$. Moreover, $\operatorname{det}_{5}$ has no zeros for $m^{2}>0$ for real coupling. Again, the proof of this follows immediately from the proof in Sec. III C of Ref. [1] that $\operatorname{det}_{4}$ has no zeros in $\mathrm{QED}_{3}$ for $m^{2}>0$ and real coupling. Hence, ln det $_{5}$ is also infinitely differentiable in $m^{2}$ on $(0, \infty)$ and will have an asymptotic expansion in $1 / m^{2}$ for a restricted class of fields. By Furry's theorem and power counting we know that the first term in its expansion for (anti-) self-dual fields will be $O\left(\int d^{4} x B^{6} / m^{8}\right)$.

This leaves the first term in (4.11). By inspection we now have for large $m^{2}$ and (anti-) self-dual fields

$$
\ln \operatorname{det}_{\mathrm{QED}_{4}}=\frac{1}{60 \pi^{2} m^{2}} \int d^{4} x\left(\partial_{\mu} B\right)^{2}+R_{2}+R_{4}+R_{5}
$$

Here $R_{2}$ is the remainder from the second-order term which is of order $\int d^{4} x\left(\partial_{\mu} \partial_{\nu} B\right)^{2} / m^{4}$; $R_{4}$ is the remainder from the fourth-order term and is of order $\int d^{4} x B^{4} / m^{4}$, and $R_{5}$, the remainder from ln $\operatorname{det}_{5}$, is of order $\int d^{4} x B^{6} / m^{8}$. Therefore, provided $A_{\mu} \in L^{n}\left(\mathbb{R}^{4}\right), n>4$, and the integrals of $\left(\partial_{\mu} B\right)^{2},\left(\partial_{\mu} \partial_{\nu} B\right)^{2}, B^{4}$ and $B^{6}$ are finite, ln $\operatorname{det}_{\mathrm{QED}_{4}}$ becomes positive before dropping off to zero. This establishes that $\ln \operatorname{det}_{\mathrm{QED}_{4}}$ has at least one zero for $m^{2}>0$, provided $\mathbf{B}$ (and hence $\mathbf{E}$ ) satisfy the above conditions and (4.8) is valid.

The existence and location of a mass zero in $\ln \operatorname{det}_{\mathrm{QED}_{4}}$ is renormalization dependent. The connection between different renormalizations is simple: if instead of subtracting at $k^{2}=0$ subtraction is made at $k^{2}=\lambda^{2},(2.1)$ or 4.11 give

$$
\begin{aligned}
\ln \operatorname{det}_{\mathrm{QED}_{4}}\left(m^{2}, \lambda^{2}\right)= & \ln \operatorname{det}_{\mathrm{QED}_{4}}\left(m^{2}, 0\right) \\
& +\frac{\|F\|^{2}}{8 \pi^{2}} \int_{0}^{1} d z z(1-z) \ln \left(\frac{m^{2}}{z(1-z) \lambda^{2}+m^{2}}\right) .
\end{aligned}
$$


This trivial shift in the value of $\ln \operatorname{det}_{\mathrm{QED}_{4}}$ shows that a mass zero of the experimentally relevant determinant $\ln \operatorname{det}_{\mathrm{QED}_{4}}\left(m^{2}, 0\right)$ causes $\ln \operatorname{det}_{\mathrm{QED}_{4}}\left(m^{2}, \lambda^{2}\right)$ to reduce to a simple quadratic in the field strength.

\section{ACKNOWLEDGEMENTS}

The author wishes to thank Gerald Dunne for helpful comments on duality transformations. 


\section{REFERENCES}

[1] M. P. Fry, Phys. Rev. D 54, 6444 (1996).

[2] M. P. Fry, Phys. Rev. D 45, 682 (1992); D 47, 743(E) (1993).

[3] G. Dunne and T. Hall, Phys. Rev. D 58, 105022 (1998).

[4] G. Dunne and T. Hall, Phys. Rev. D 60, 065002 (1999).

[5] M. P. Fry, Phys. Rev. D 51, 810 (1995).

[6] I. Aitchison and C. Fraser, Phys. Rev. D 31, 2605 (1985).

[7] H.W. Lee, P.Y. Pac, and H.K. Shin, Phys. Rev. D 40, 4202 (1989).

[8] D. Cangemi, E. D’Hoker, and G. Dunne, Phys. Rev. D 51, R2513 (1995).

[9] V.P. Gusynin and I.A. Shovkovy, Can. J. Phys. 74, 282 (1996).

[10] V.P. Gusynin and I.A. Shovkovy, J. Math. Phys. 40, 5406 (1999).

[11] E. Brezin and C. Itzykson, Phys. Rev. D 2, 1191 (1970);

C. Martin and D. Vautherin, Phys. Rev. D 38, 3593 (1988).

[12] E. Seiler, Phys. Rev. D 22, 2412 (1980).

[13] E. Seiler, Gauge Theories: Fundamental Interactions and Rigorous Results, Proceedings of the International School of Theoretical Physics, Poiana Brasov, Romania, 1981, edited by P. Dita, V. Georgescu, and P. Purice, Progress in Physics Vol. 5 (Birkhäuser, Boston 1982), p. 263.

[14] E. Seiler, Gauge Theories as a Problem of Constructive Quantum Field Theory and Statistical Mechanics, Lecture Notes in Physics Vol. 159 (Springer, Berlin/Heidelberg/New York, 1982).

[15] J. Schwinger, Phys. Rev. 128, 2425 (1962).

[16] Y. Aharonov and A. Casher, Phys. Rev. A 19, 2461 (1979).

[17] M. P. Fry, Phys. Rev. D 53, 980 (1996). 
[18] J. Schwinger, Phys. Rev. 82, 664 (1951).

[19] M. P. Fry, J. Math. Phys. 41, 1691 (2000).

[20] D. Brydges, J. Fröhlich, and E. Seiler, Ann. Phys. (N.Y.) 121, 227 (1979).

[21] D. H. Weingarten, Ann. Phys. (N.Y.) 126, 154 (1980).

[22] M. P. Fry, Phys. Rev. D 55, 968 (1997); D 56, 6714(E), (1997).

[23] N. Dunford and J. T. Schwartz, Linear Operators, Part II (Interscience, New York, 1963).

[24] E. Seiler, Commun. Math. Phys. 42, 163 (1975).

[25] B. Simon, Adv. Math. 24, 244 (1977).

[26] B. Simon, Trace Ideals and their Applications, London Mathematical Society Lecture Notes Series 35 (Cambridge University Press, Cambridge, England, 1979).

[27] E. Seiler and B. Simon, Commun. Math. Phys. 45, 99 (1975).

[28] I. C. Gohberg and M. G. Kreǐn, Introduction to the Theory of Linear Nonselfadjoint Operators, Translations of Mathematical Monographs Vol. 18 (American Mathematical Society, Providence, 1969), p. 163.

[29] W. B. Ford, Studies on Divergent Series and Summability and the Asymptotic Developments of Functions Defined by Maclaurin Series (Chelsea, New York, 1960), p. 30.

[30] H. Euler and B. Kockel, Naturwissenschaften 23, 246 (1935); W. Heisenberg and H. Euler, Z. Phys. 98, 714 (1936); V. Weisskopf, K. Dan. Vidensk. Selsk. Mat. Fys. Medd. 14, $6(1936)$.

[31] M. Atiyah and I. Singer, Ann. Math. 87, 484 (1968); 87, 546 (1968); M. Atiyah, R. Bott, and V. Patodi, Invent. Math. 19, 279 (1973).

[32] R. Musto, L. O’Raifeartaigh, and A. Wipf, Phys. Lett. B 175, 433 (1986).

[33] R. Karplus and M. Neuman, Phys. Rev. 80, 380 (1950). 